Supporting Information for:

\title{
Morphological Evolution of Tetrachlorinated Perylene Bisimides with Lengthy Alkyl Substituent Polycrystalline Thin Films during Reversible Phase Transitions
}

Rui Xin, ${ }^{a}$ Shaojuan Wang, ${ }^{a}$ Cheng Zeng, ${ }^{b}$ Andong Ji, ${ }^{c}$ Jie Zhang,${ }^{c}$ Zhongjie Ren, ${ }^{* c}$ Wei Jiang, ${ }^{b}$ Zhaohui Wang, ${ }^{* b}$ and Shouke Yan*a,c

a.Key Laboratory of Rubber-Plastics, Ministry of Education, Qingdao University of Science \& Technology, Qingdao 266042, China. E-mail: skyan@mail.buct.edu.cn

${ }^{b}$ Key Laboratory of Organic Solids, Beijing National Laboratory for Molecular Sciences, Institute of Chemistry, Chinese Academy of Sciences, Beijing 100190, China. E-mail: wangzhaohui@iccas.ac.cn ${ }^{c}$.State Key Laboratory of Chemical Resource Engineering, Beijing University of Chemical Technology Beijing, 100029, China. E-mail: renzj@mail.buct.edu.cn

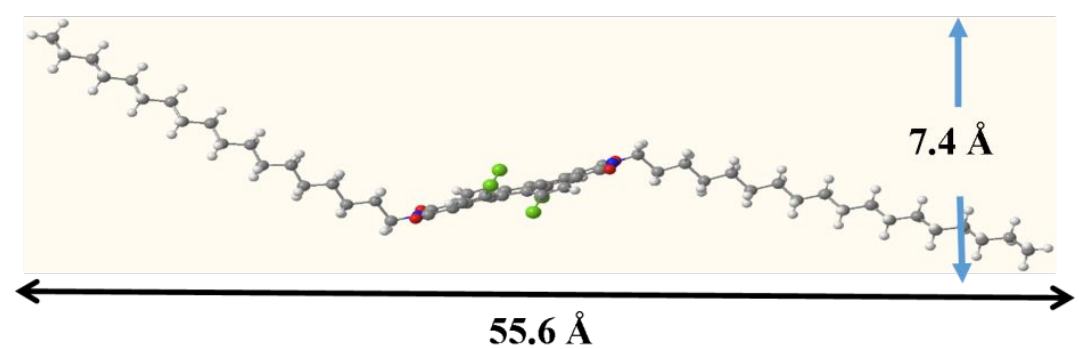

Figure S1. Molecular structure of C18-4C1PBI obtained by energy optimization through

Materials Studio software. 

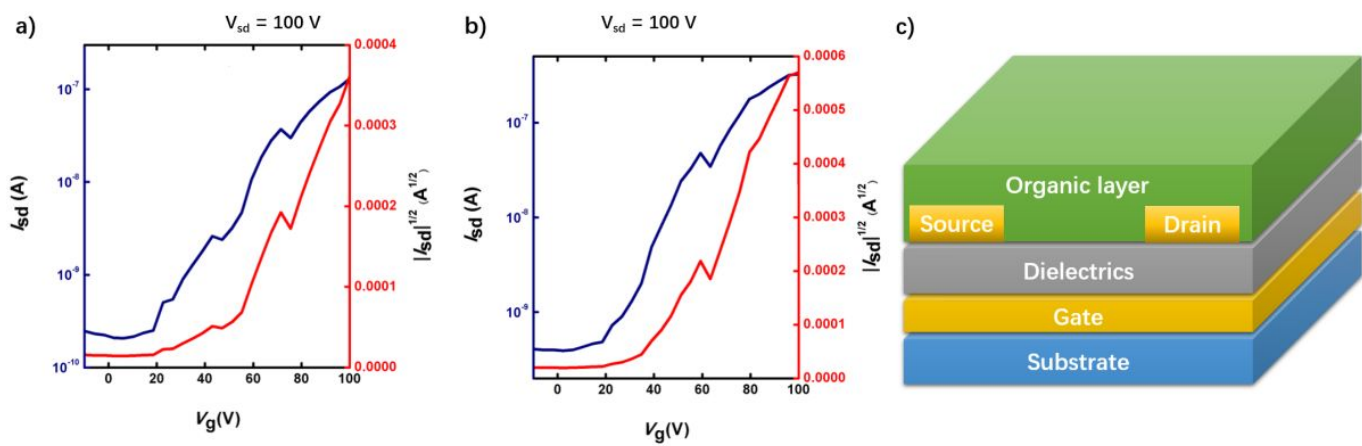

Figure S2. Transfer characteristics of a thin-film transistor based on C18-4ClPBI at a) $100{ }^{\circ} \mathrm{C}$ and b) $120^{\circ} \mathrm{C}$. c) Schematic diagram of bottom-gate and bottom-contact OFET. 